\title{
Spontaneous coronary dissection presenting with reperfusion arrhythmia: A case report
}

\author{
Khawar Maqsood $^{1^{*}}$, Marwan Badri ${ }^{2}$, James F. Burke ${ }^{3}$, Wajeeha Saeed ${ }^{4}$, Ghazi Mirrani ${ }^{2}$, \\ Nosheen Sarwar ${ }^{2}$, Joseph Kusick ${ }^{2}$, Frank C. McGeehin ${ }^{3}$, Paul M. Coady ${ }^{3}$, Muhammad R. Sardar ${ }^{3}$ \\ ${ }^{1}$ Department of Medicine, Baystate Medical Center, Tufts University School of Medicine, Springfield, USA; \\ *Corresponding Author: dockmaq@gmail.com \\ ${ }^{2}$ Department of Medicine, Division of Internal Medicine, Lankenau Medical Center, Wynnewood, USA \\ ${ }^{3}$ Department of Medicine, Division of Cardiology, Lankenau Medical Center, Wynnewood, USA \\ ${ }^{4}$ Department of Medicine, Division of Internal Medicine, Bronx-Lebanon Hospital Center, New York, USA
}

Received 19 March 2013; revised 22 April 2013; accepted 15 May 2013

Copyright (C) 2013 Khawar Maqsood et al. This is an open access article distributed under the Creative Commons Attribution License, which permits unrestricted use, distribution, and reproduction in any medium, provided the original work is properly cited.

\section{ABSTRACT}

Spontaneous coronary dissection is more commonly reported in females and is an important differential diagnosis for acute coronary syndrome. Accelerated idioventricular rhythm has been reported before with reperfusion post myocardial ischemia. We report a case of accelerated idioventricular rhythm in a patient with spontaneous coronary artery dissection. A 45-yearold Caucasian female presented with left sided chest pain radiating to the neck and palpitations. Admission ECG showed accelerated idioventricular rhythm. Troponin I peaked at $0.5 \mathrm{ng} / \mathrm{ml}$. Coronary angiography showed mid to distal left anterior descending artery dissection with adequate distal flow. Patient was initially medically managed with aspirin, metoprolol, intravenous heparin and eptifibatide infusions but continued to have symptoms of unstable angina. She underwent successful percutaneous coronary intervention with 2 drug eluting stents and was discharged back home symptom free on dual platelet therapy. Spontaneous coronary artery dissection is an important differential diagnosis for acute coronary syndrome especially in younger females. Accelerated idioventricular rhythm can be a presentation of coronary dissection and may indicate instability. Early percutaneous coronary intervention should be considered in such patients.

Keywords: Spontaneous Coronary Dissection; Accelerated Idioventricular Rhythm

\section{INTRODUCTION}

Spontaneous Coronary Artery Dissection (SCAD) is more commonly reported in female patients [1]. Alongside coronary vasospasm and takotsubo cardiomyopathy, it is an important differential diagnosis of ACS in females of this age group, in whom atherosclerotic coronary artery disease is rare. SCAD can present in variable ways that span the spectrum of acute coronary syndromes, including unstable angina, acute ST-elevation myocardial infarction, ventricular tachycardia and sudden death. The patient in this report had a clinical presentation of ACS that was uniquely associated with accelerated idioventricular rhythm (AIVR). Although AIVR can be seen in several conditions including subarachnoid hemorrhage, digitalis toxicity, hyperkalemia and in children with no heart disease [2], it is most commonly seen in the context of myocardial infarction. While it used to be considered a sign of successful reperfusion following thrombolysis for acute myocardial infarction [3], a more recent report suggests its presence is an indication of myocardial injury and delayed microvascular reperfusion [4].

\section{CASE PRESENTATION}

A 45-year-old Caucasian female presented with sudden onset, left sided pressure-like chest pain radiating to the neck and associated with palpitations. This was preceded by an emotional happy situation of her family reunion. Her medical history is significant for atrioventricular nodal reentry tachycardia (AVNRT) treated with radiofrequency ablation 3 years prior to this presentation. On arrival in the emergency room the patient was free of chest pain. Her blood pressure was 172/99 mmHg and heart rate was 94/minute. An ECG obtained on arrival 
showed a wide complex rhythm with evidence of atrioventricular dissociation consistent with accelerated idioventricular rhythm (AIVR) (Figure 1: AIVR. Black arrows indicating AV dissociation. Ventricular rate of $94 / \mathrm{min})$. Several minutes later her rhythm changed and a repeat ECG showed sinus rhythm with subtle T-inversion in lead V2 and no ST changes. Troponin I peaked at 0.5 $\mathrm{ng} / \mathrm{ml}$. Her pregnancy test was negative. Acute coronary syndrome was suspected and she was treated with aspirin, metoprolol, intravenous heparin and eptifibatide infusions. Coronary catheterization showed tapering of the mid to distal LAD before it re-expands and wraps around the apex, suggestive of a diagnosis of coronary dissection (Figures 2(a) and (b): White arrows indicate the segment of coronary artery dissection involving LAD). All other coronary arteries were free of significant disease. Given the absence of symptoms and adequate distal flow no intervention was undertaken at that time, and the patient was managed medically while being observed in the coronary care unit. In the following 48 hours, she had intermittent chest pain at rest and chest pressure on mild to moderate exertion. As the clinical picture was consistent with unstable angina, percutaneous coronary intervention of the dissecting lesion was performed using two drug eluting stents with satisfactory angiographic result and TIMI-3 flow (Figure 3(a): Attempted wire with balloon and stenting of dissection segment. Figure 3(b): TIMI-3 flow post stenting LAD stents). The patient's symptoms completely resolved afterwards and she was discharged home on dual antiplatelet therapy.

\section{DISCUSSION}

Since SCAD was first described over 80 years ago, a large number of cases have been reported in the literature. Over $75 \%$ of reported cases (including this case) involved the $\mathrm{LAD}[5,6]$. The predominant involvement of the LAD is unexplained. SCAD leads to acute coronary syndrome due to compression of the true coronary lumen by the false lumen resulting from dissection. The dissecttion may also involve the origin of a branch coronary artery causing complete occlusion of that vessel. Either way, ischemia distal to the area of dissection ensues. The exact pathogenesis behind the dissection process is still unknown. Pre-menopausal females in general and peripartum females in particular have higher than average incidence of SCAD [1]. Other correlations have also been made between SCAD and connective tissue diseases $[7,8]$, vasculitides $[9,10]$, oral contraceptive [11] and cocaine use [12].

Non-invasive medical management is considered an acceptable strategy in patients with distal disease, small branch dissection and when TIMI-3 flow is seen distal to the dissection $[13,14]$ and conservatively-treated patients have been shown to have good prognosis [15]. It is understandable that efforts should be made to avoid PCI in young patients given the likelihood of future in-stent restenosis, particularly when large area of stenting is involved as in the case our patient. Moreover, PCI for coronary dissection poses several technical challenges and can lead to worsening of the dissection if the guide

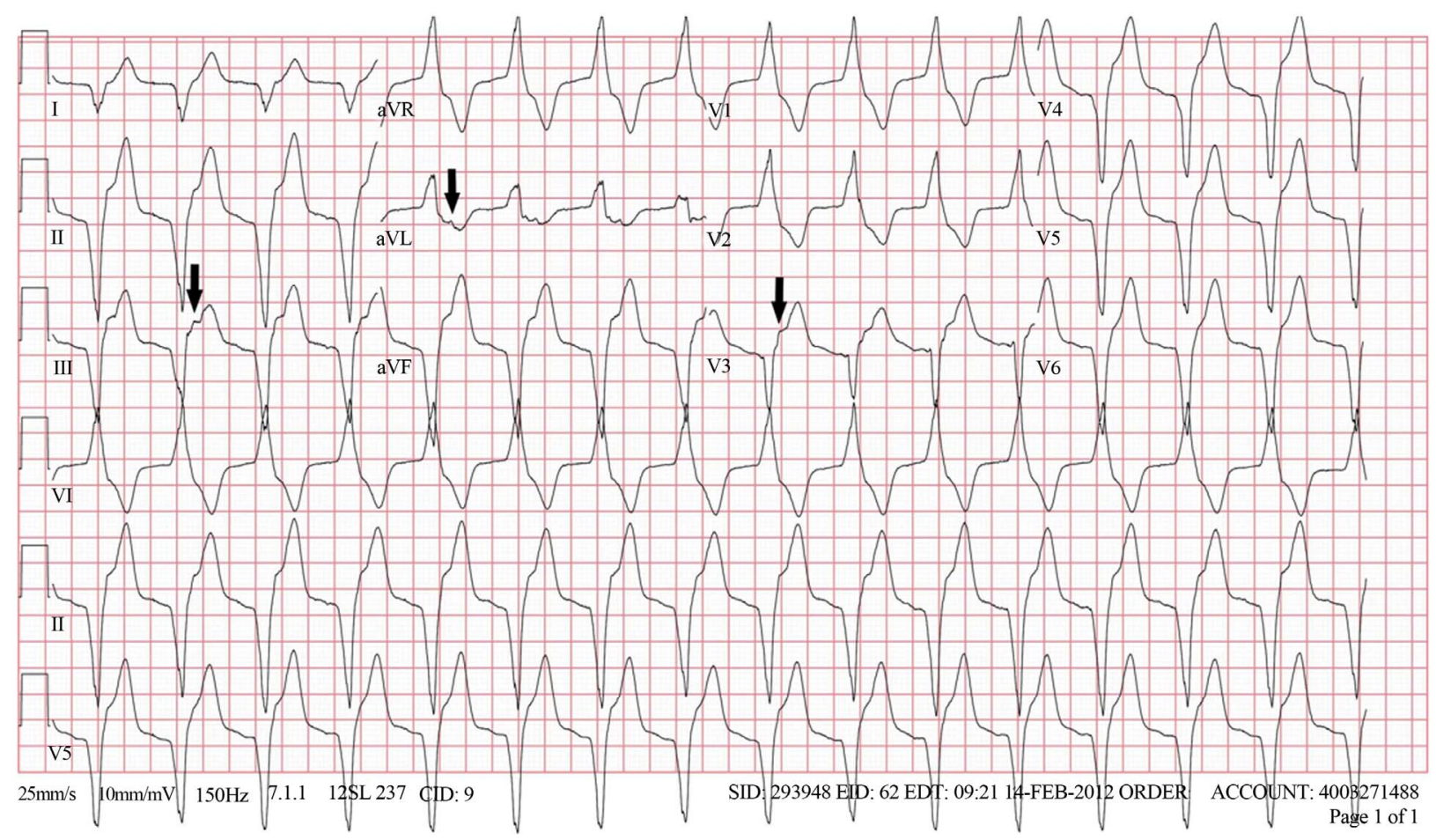

Figure 1. AIVR. Black arrows indicating AV dissociation. Ventricular rate of 94/min. 


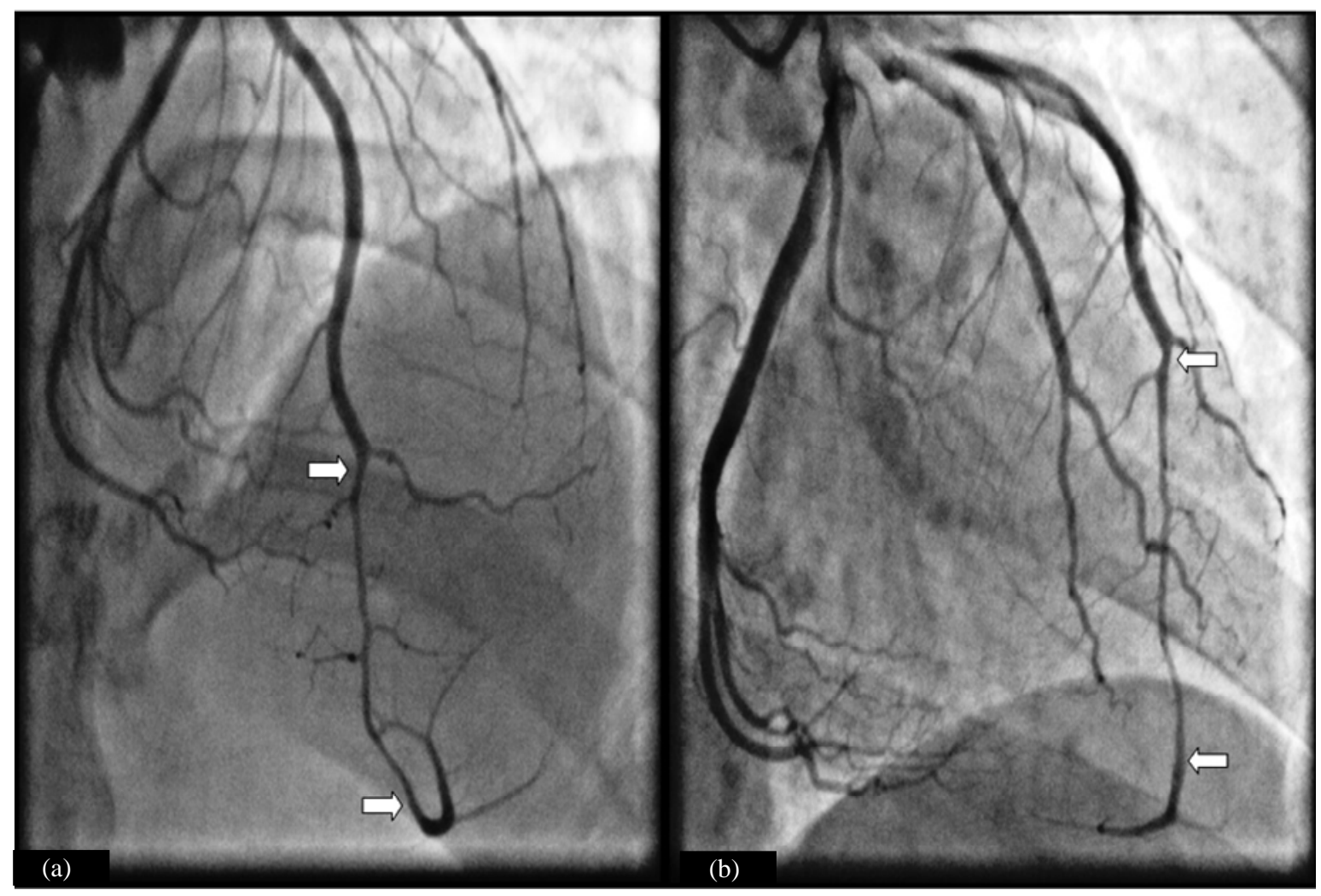

Figure 2. White arrows indicate the segment of coronary artery dissection involving LAD.

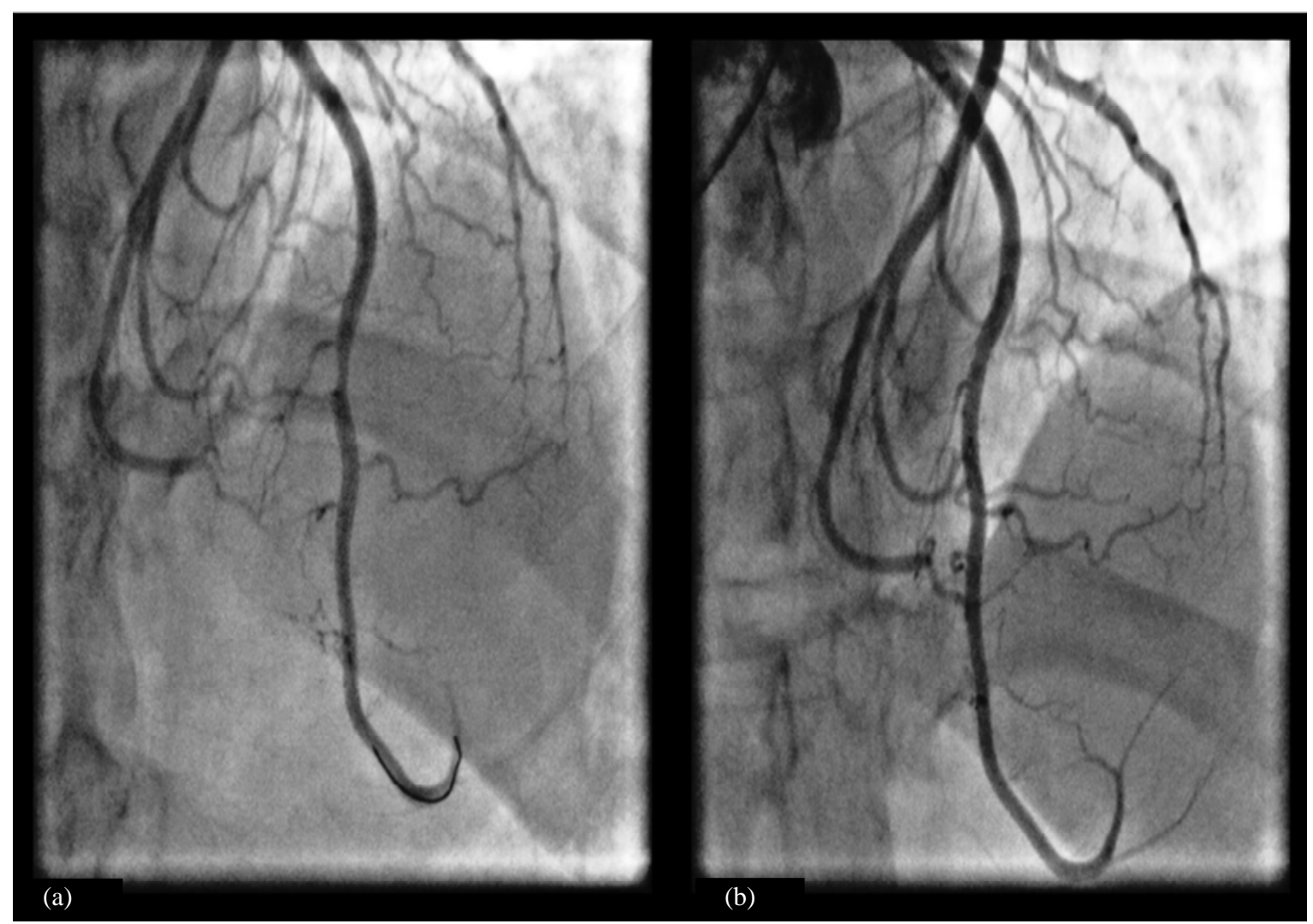

Figure 3. (a) Attempted wire with balloon and stenting of dissection segment; (b) TIMI 3 flow post stenting LAD stents. 
wire enters the false lumen, which can lead to propagation of the intramural hematoma. However, when large vessels are involved with clinical and electrocardiographic features of unstable disease are present, early invasive management should be attempted. This case represents a mixed presentation between the two extremes of the disease. The patient was hemodynamically stable at the time of presentation and had no major residual ECG abnormalities. Coronary angiography showed normal TIMI-3 flow distal to the lesion, therefore initially conservative management was adopted. The clinical course following that, with symptoms of unstable angina indicated the need for percutaneous coronary intervention.

\section{CONCLUSION}

SCAD is one of the leading differential diagnoses in young females presenting with acute coronary syndrome. The presentation of SCAD is variable and includes AIVR. This may indicate instability and therefore early percutaneous coronary intervention should be considered. There is need for more research to establish a standard of care in SCAD.

\section{REFERENCES}

[1] Shamloo, B.K., Chintala, R.S., Nasur, A., Ghazvini, M., Shariat, P., Diggs, J.A. and Singh, S.N. (2010) Spontaneous coronary artery dissection: Aggressive vs. conservative therapy. Journal of Invasive Cardiology, 22, 222-228.

[2] Comerford, T.J. and Propert, D.B. (1979) Accelerated idioventricular rhythm in patients without acute myocardial infarction. Angiology, 30, 768-775. doi:10.1177/000331977903001106

[3] Grimm, W., Hoffmann, J. and Maisch, B. (1994) Accelerated idioventricular rhythm. Zeitschrift für Kardiologie, 83, 898-907.

[4] Terkelsen, C.J., Sorensen, J.T., Kaltoft, A.K., Nielsen, S.S., Thuesen, L., Botker, H.E. and Lassen, J.F. (2009) Prevalence and significance of accelerated idioventricular rhythm in patients with ST-elevation myocardial infarction treated with primary percutaneous coronary intervention. American Journal of Cardiology, 104, 1641-1646. doi:10.1016/j.amjcard.2009.07.037

[5] Jorgensen, M.B., Aharonian, V., Mansukhani, P. and Mahrer. P.R. (1994) Spontaneous coronary dissection: A cluster of cases with this rare finding. American Heart Journal, 127, 1382-1387.

\section{doi:10.1016/0002-8703(94)90060-4}

[6] Hering, D., Piper, C., Hohmann, C., Schultheiss, H.P. and Horstkotte, D. (1998) Incidence, etiology and therapy of spontaneous coronary artery dissection. A prospective monocenter study of 3800 consecutive patients. Zeitschrift für Kardiologie, 87, 961-970. doi:10.1007/s003920050253

[7] Bateman, A.C., Gallagher, P.J. and Vincenti, A.C. (1995) Sudden death from coronary artery dissection. Journal of Clinical Pathology, 48, 781-784. doi:10.1136/jcp.48.8.781

[8] Ades, L.C., Waltham, R.D., Chiodo, A.A. and Bateman, J.F. (1995) Myocardial infarction resulting from coronary artery dissection in an adolescent with Ehlers-Danlos syndrome type IV due to a type III collagen mutation. British Heart Journal, 74, 112-116. doi:10.1136/hrt.74.2.112

[9] Aldoboni, A.H., Hamza, E.A., Majdi, K., et al. (2002) Spontaneous dissection of coronary artery treated by primary stenting as the first presentation of systemic lupus erythematosus. Journal of Invasive Cardiology, 14, 694696.

[10] Chu, K.H., Menapace, F.J., Blankenship, J.C., et al. (1998) Polyarteritis nodosa presenting as myocardial infarction with coronary dissection. Catheterization and Cardiovascular Diagnosis, 44, 320-324.

doi:10.1002/(SICI)1097-0304(199807)44:3<320::AID-C CD16>3.3.CO;2-B

[11] Evangelou, D., Letsas, K.P., Korantzopoulos, P., Antonellis, I., Sioras, E. and Kardaras, F. (2006) Spontaneous coronary artery dissection associated with oral contraceptive use: A case report and review of the literature. International Journal of Cardiology, 112, 380-382. doi:10.1016/j.ijcard.2005.07.069

[12] Steinhauer, J.R. and Caulfield, J.B. (2001) Spontaneous coronary artery dissection associated with cocaine use: a case report and brief review. Cardiovascular Pathology, 10, 141-145. doi:10.1016/S1054-8807(01)00074-6

[13] Adlam, D., Cuculi, F., Lim, C. and Banning, A. (2010) Management of spontaneous coronary artery dissection in the primary percutaneous coronary intervention era. Journal of Invasive Cardiology, 22, 549-553.

[14] Kamran, M., Guptan, A. and Bogal, M. (2008) Spontaneous coronary artery dissection: case series and review. Journal of Invasive Cardiology, 20, 553-559.

[15] Zampieri, P., Aggio, S., Roncon, L., Rinuncini, M., Canova, C., Zanazzi, G., Fiorencis, R. and Zonzin, P. (1996) Follow up after spontaneous coronary artery dissection: A report of five cases. Heart, 75, 208-209. doi:10.1136/hrt.75.2.206 